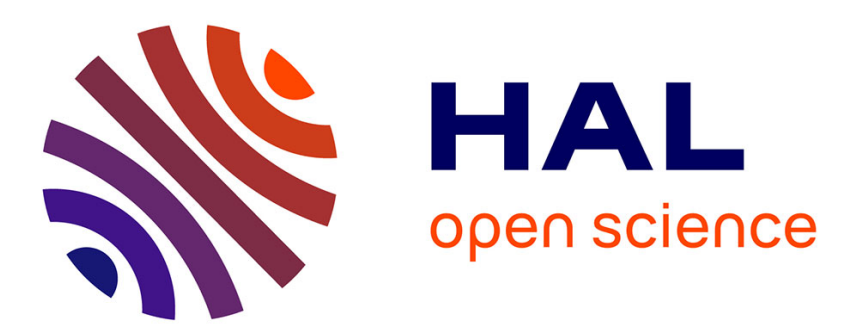

\title{
Nonlinear model reduction for computational vibration analysis of structures with weak geometrical nonlinearity coupled with linear acoustic liquids in the presence of linear sloshing and capillarity
}

Roger Ohayon, Christian Soize

\section{To cite this version:}

Roger Ohayon, Christian Soize. Nonlinear model reduction for computational vibration analysis of structures with weak geometrical nonlinearity coupled with linear acoustic liquids in the presence of linear sloshing and capillarity. Computers and Fluids, 2016, 141, pp.82-89.

10.1016/j.compfluid.2016.03.032 . hal-01296500v2

\author{
HAL Id: hal-01296500 \\ https://hal.science/hal-01296500v2
}

Submitted on 29 Mar 2019

HAL is a multi-disciplinary open access archive for the deposit and dissemination of scientific research documents, whether they are published or not. The documents may come from teaching and research institutions in France or abroad, or from public or private research centers.
L'archive ouverte pluridisciplinaire HAL, est destinée au dépôt et à la diffusion de documents scientifiques de niveau recherche, publiés ou non, émanant des établissements d'enseignement et de recherche français ou étrangers, des laboratoires publics ou privés. 


\title{
Nonlinear model reduction for computational vibration analysis of structures with weak geometrical nonlinearity coupled with linear acoustic liquids in the presence of linear sloshing and capillarity
}

\author{
Roger Ohayon ${ }^{\mathrm{a}}$, Christian Soize ${ }^{\mathrm{b}, *}$ \\ ${ }^{a}$ Structural Mechanics and Coupled Systems Laboratory, Conservatoire National des Arts et \\ Métiers (CNAM), 2 rue Conté, 75003 Paris, France \\ ${ }^{b}$ Université Paris-Est, Laboratoire Modélisation et Simulation Multi-Echelle, MSME UMR 8208 \\ CNRS, 5 bd Descartes, 77454 Marne-La-Vallée, Cedex 2, France
}

\begin{abstract}
This paper deals with a novel formulation of a nonlinear reduced-order computational model for analyzing the nonlinear vibrations of a linear viscoelastic structure with weak nonlinear geometrical effects, coupled with a linear acoustic liquid with sloshing and capillarity on the free surface. The model proposed is derived from the one used in fluid-structure interaction for linear systems, for which the analysis of the acoustic-sloshing-capillarity phenomena is efficient thanks to the use of a projection on the linear modes of the linear acoustic liquid and on the sloshing modes with capillarity. Concerning the construction of the vector basis for the structure, it is proposed to use a POD approach for the viscoelastic structure with weak nonlinear geometrical effects and taking into account the added mass induced by the liquid. The methodology for constructing the vector bases of the admissible sets and for obtaining the nonlinear ROM are detailed. The computational nonlinear ROM that is presented can directly be used for analyzing the vibrations of such fluid-structure systems using commercial finite element softwares for computing the vector bases and the projections.
\end{abstract}

Keywords: Nonlinear vibration, nonlinear reduced-order model, ROM, structure coupled with liquid, FSI, geometrical nonlinearity, sloshing, capillarity, surface

\footnotetext{
${ }^{*}$ Corresponding author: C. Soize, christian.soize@ univ-paris-est.fr

Email addresses: roger. ohayon@ cnam. fr (Roger Ohayon), christian.soize@univ-paris-est.fr(Christian Soize )
} 
tension,

\section{Introduction}

This paper is the continuation of the work published in [1] for which the linear behavior of the structure is replaced by a weak geometrical nonlinear behavior (a requirement being that if the structural nonlinear term goes to zero, the linear formulation is retrieved). The structure contains a linear dissipative acoustic liquid (no flow), with linear sloshing and capillarity, and for which the effects of internal gravity waves are neglected. A linear formulation for the acoustic liquid is kept in order to preserve the vibration analysis expressed in terms of acoustic and sloshing modes for small (infinitesimal) fluid displacements (consequently, the ALE formulation that requires a mesh deformation is not well adapted to such a requirement). Since the structure can undergo small finite displacements, the model proposed consists in writing the equations of the linear acoustic fluid on the undeformed configuration and in transporting the actual configuration of the structure on its undeformed configuration. In addition, we also want to preserve a slip condition for the fluid on the fluid-structure interface, which is facilitated with the formulation proposed (such a condition is of course not valid in microand nano-fluidics). It should be noted that the underlying hypotheses of such a formulation (small finite displacements for the structure and linear behavior of the acoustic liquid in presence of sloshing and capillarity effects) are justified in many applications (see for instance the experimental observations described in [2]). The simplifications introduced in the formulation proposed (such as the noninclusion of the finite fluctuations of the wetting fluid-structure interface) introduce uncertainties induced by modeling errors. Such uncertainties could be taken into account in a probabilistic framework of uncertainty quantification. Since these types of uncertainties are not relevant of the usual parametric probabilistic approach [3], an open way of research could be the nonparametric probabilistic approach of modeling errors $[4,5]$. But, for the present fluid-structure interaction problem, novel nonparametric approaches should be developed in order to take into account modeling errors in computational fluid dynamics.

The novel feature of the paper is to propose the construction of a computational nonlinear reduced-order model (ROM) derived from the one used in linear fluid-structure interaction (for which the analysis of the acoustic-sloshingcapillarity phenomena is efficient thanks to the use of a projection on the linear modes of the acoustic liquid and on the sloshing modes with capillarity), in taking 
into account weak geometric nonlinearities in the structure. Furthermore, for the structure with geometric nonlinearities, a linear viscoelastic constitutive equation without memory effects is used for generating damping in the structure instead of the use of an empirical damping model that is arbitrary added in the reduced-order equations (as frequently done).

Concerning the linear formulation for the compressible liquid with free surface, the sloshing is taken into account with surface tension (capillarity) effects. We refer the reader to $[6,7]$ for the classical theory on capillarity, to $[8,9,10]$ for developments of the behavior of liquids in microgravity environment, to $[11,12]$ for general analyzes of sloshing problems for incompressible liquids in rigid structures, to $[13,14,15,16,17,18]$ for sloshing problems of incompressible liquids without capillarity effects in elastic structures, to [13, 19, 20, 21] for sloshing problems of incompressible liquids with capillarity effects in rigid structures, to $[22,23,24,25,26,27,28,13,29]$ for the conditions of contact angle between the free surface and the structure, to [30] for sloshing problems of incompressible liquids with capillarity effects in elastic structures, to [31, 32, 33] for sloshing problems of compressible liquids with capillarity effects in rigid structures, to [1] for linear dissipative acoustic liquids with sloshing and capillarity effects in linear elastic structures. Concerning nonlinear sloshing and capillarity for incompressible liquids in rigid tanks submitted to rigid body motions, see [34, 35, 36, 37, 38]. Concerning computational reduced-order models for the linear vibration of structures containing compressible liquids without surface tension and without sloshing effects, we refer the reader for instance to $[13,39,40,41,5]$. Note that the case of a structure with weak geometrical nonlinearities coupled with a linear acoustic fluid has been investigated in the high-frequency domain in [42].

General computational methods for strongly nonlinear fluid-structure interaction problems including sloshing and capillarity, without the introduction of reduced-order models (but generally using ALE-based approach for the fluid), can be found in $[43,44,45,46,47,48,49,50,51,52,53,54,55]$. A general discussion on elasto-capillary fluid-structure interaction including fluid-solid surface-tension effects and taking into account a transition layer made up a diffuse-interface model related to a phase separation between complex fluids and/or soft matters (CahnHilliard model [56]) can be found in [57].

For constructing computational reduced-order model (ROM) in linear and nonlinear structural dynamics and in fluid-structure interaction, some method- 
ologies have extensively been analyzed in literature. A lot of works have been published for constructing ROM and parametric ROM for linear and nonlinear computational models in dynamics in the area of computational structural dynamics and fluid-structure interaction among which [13, 58, 59, 60, 61, 62, 63, 64, 65] and in vibroacoustics $[40,66,67,5]$. A hyper reduction is required for reducing the numerical cost of the ROM construction (see for instance [68, 69, 70, 71]). The construction of the reduced-order basis (ROB) is based on the computation of solution snapshots performed with the computational model. The Proper Orthogonal Decomposition (POD) method [72] is a possible approach for constructing the ROB as used in linear structural dynamics for all possible input forces [73] or for a given set of input forces [74, 75, 76, 77]. When the POD method is used for constructing the ROB for a nonlinear computational model $[62,78]$, a large number of snapshots is used (for time evolution problems, the time becomes a parameter) and the responses are compressed into a low-dimensional ROB using, for example, the singular value decomposition method and several ingredients such as those presented in $[79,80]$.

In Section 2, we give the notations and hypotheses used. Section 3 deals with the nonlinear equation for the structure expressed in the reference configuration. The boundary value problem for the nonlinear fluid-structure problem is presented in Section 4 for which the formulation retained is expressed using the pressure field in the liquid, the elevation of the free surface, and the displacement field of the structure. In Sections 5 and 6, we present the computational fluidstructure model. The decomposition of the admissible space of the discretized coupled problem and the construction of the associated vector bases are presented in Section 7. Finally, the nonlinear reduced-order computational model is detailed in Section 9.

\section{Notations and hypotheses}

We consider the fluid-structure system in its reference configuration defined in Figure 1. For this reference configuration, the structure is undeformed and occupies an open, bounded, and connected subset $\Omega_{S}$ of $\mathbb{R}^{3}$. The existing prestresses are not taken into account, which means that the undeformed configuration corresponds to a natural state. In the reference configuration, the undeformed structure contains a liquid, which occupies an open and bounded domain $\Omega_{L}$. The geometry of domain $\Omega_{L}$ results from a pre-computation for finding the static equilibrium of the liquid in the structure considered as rigid, and submitted to the gravity ef- 
fects and to the surface-tension effects (capillarity) of the free surface and of the fluid-structure interface. Since the liquid is linear and since we are interested in studying the dynamics around the static equilibrium, the fluid in domain $\Omega_{L}$ is assumed to be in a natural state.

The constitutive equation for the structure is assumed to be linear viscoelastic in finite displacements with instantaneous memory, described by a displacement field measured from the reference configuration, and can undergo weak finite displacements (small strain and moderate rotations). The liquid is a linear dissipative acoustic fluid, which means that the liquid is homogeneous, compressible, inviscid with a volumic additional dissipative term, described by a pressure field, and which is in irrotational infinitesimal motions with respect to the reference configuration. It should be noted that the liquid is inviscid on the fluid-structure interface on which a slip condition will be written.

The physical space is referred to a cartesian reference system. In the reference configuration, the boundary of $\Omega_{L}$ is assumed to be smooth enough and is written as $\partial \Omega_{L}=\Gamma_{L} \cup \gamma \cup \Gamma$. The open part $\Gamma_{L}$ is the fluid-structure interface. The open part $\Gamma$ is the free surface of the liquid in its reference configuration. The close part $\gamma$ is a curve (the contact line), which is such that $\gamma=\partial \Gamma_{L}=\partial \Gamma$. The boundary of $\Omega_{S}$ (supposed smooth enough) is written as $\partial \Omega_{S}=\Gamma_{E} \cup \Gamma_{L} \cup \gamma \cup \Gamma_{G}$ (see Figure 1). The boundary of the open parts $\Gamma_{L}$ and $\Gamma_{G}$ is the curve $\gamma$. The domain whose boundary is the open set $\Gamma \cup \Gamma_{G}$ is empty or is filled by a gas whose effects are neglected for sake of brevity (this is the domain above $\Omega_{L}$ in Figure 1). The external unit normal to $\partial \Omega_{S}$ is denoted as $\mathbf{n}^{S}$ while the one to $\partial \Omega_{L}$ is denoted as n. The external unit normal to $\gamma$ belonging to the tangent plane to surface $\Gamma$ is denoted as $\nu$. Let $\Omega_{S}(t)$ be the actual domain occupied by the structure at time $t$ and let $\partial \Omega_{S}(t)$ be its boundary.

We are interested in analyzing the nonlinear vibration (structural geometric nonlinearities) of the coupled system around its reference configuration. The structure is assumed to be free (free-free structure for which no prescribed Dirichlet boundary conditions are given on $\partial \Omega_{S}(t)$ ). The structure is submitted to given forces, which are applied to $\Omega_{S}(t)$, and which are assumed to be in equilibrium (in the actual configuration). 


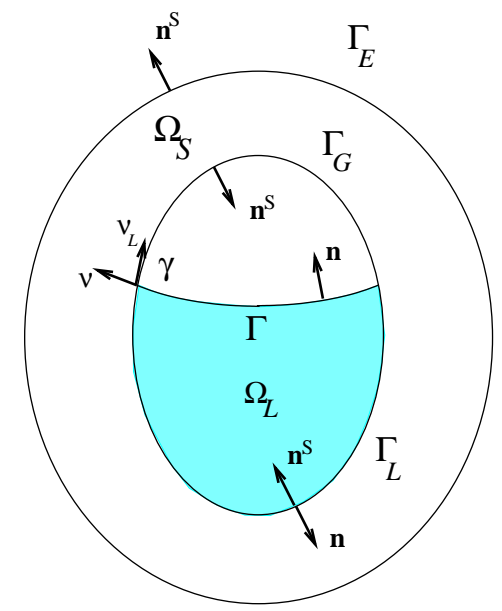

Figure 1: Reference configuration of the fluid-structure system

\section{Nonlinear equation for the structure expressed in the reference configura- tion}

In the cartesian reference system, let $\mathbf{x}=\left(x_{1}, x_{2}, x_{3}\right)$ be the position vector of a point in the reference configuration $\bar{\Omega}_{S}=\Omega_{S} \cup \partial \Omega_{S}$. In the actual (deformed) configuration $\Omega_{S}(t)$ at time $t$, the position vector $\mathbf{X}=\left(X_{1}, X_{2}, X_{3}\right)$ of the point that is transformed from $\mathbf{x}$, is written as

$$
\mathbf{X}(\mathbf{x}, t)=\mathbf{x}+\mathbf{u}(\mathbf{x}, t),
$$

in which $\mathbf{u}=\left(u_{1}, u_{2}, u_{3}\right)$ is the displacement field defined in $\bar{\Omega}_{S}$. The deformation gradient tensor $\mathbb{F}(\mathbf{x}, t)$ defined in $\Omega_{S}$ by

$$
\mathbb{F}_{i j}(\mathbf{x}, t)=\frac{\partial X_{i}(\mathbf{x}, t)}{\partial x_{j}}=\delta_{i j}+\frac{\partial u_{i}(\mathbf{x}, t)}{\partial x_{j}},
$$

and is assumed to be invertible and orientation preserving for all $\mathbf{x} \in \bar{\Omega}_{S}$ and for all $t$ :

$$
J(\mathbf{x}, t) \stackrel{\text { def }}{=} \operatorname{det} \mathbb{F}(\mathbf{x}, t)>0 .
$$

The dynamic equilibrium equations of the structure at time $t$, expressed with respect to the reference configuration, are written $[81,82]$ as

$$
\rho_{\mathrm{s}} \frac{\partial^{2} \mathbf{u}}{\partial t^{2}}-\operatorname{div}(\mathbb{F} \mathbb{S})=\mathbf{b}, \forall \mathbf{x} \in \Omega_{S},
$$


where $\rho_{\mathrm{S}}(\mathbf{x})>0$ is the mass density in the reference configuration, where $\mathbb{S}$ is the second Piola-Kirchhoff symmetric stress tensor in the reference configuration, where $\operatorname{div}(\mathbb{F} \mathbb{S})$ denotes the divergence of the second-order tensor $\mathbb{F} \mathbb{S}$, and where $\mathbf{b}=\left(b_{1}, b_{2}, b_{3}\right)$ corresponds to the transport on $\Omega_{S}$ of the body force field $\mathbf{B}$ applied to deformed configuration $\Omega_{S}(t)$, which is written as

$$
\mathbf{b}(\mathbf{x}, t)=J(\mathbf{x}, t) \mathbf{B}(\mathbf{X}(\mathbf{x}, t), t) .
$$

At time $t$, let $\mathbf{F}$ be the surface force field applied to the deformed boundary $\partial \Omega_{S}(t)$ of the actual configuration. The transport of $\mathbf{F}$ on the undeformed boundary $\partial \Omega_{S}$ is denoted by $\mathbf{f}=\left(f_{1}, f_{2}, f_{3}\right)$ and is written as

$$
\mathbf{f}(\mathbf{x}, t)=\{\operatorname{det} \mathbb{F}(\mathbf{x}, t)\}\left\|\mathbb{F}(\mathbf{x}, t)^{-T} \mathbf{n}^{S}(\mathbf{x})\right\| \mathbf{F}(\mathbf{X}(\mathbf{x}, t), t) .
$$

At time $t$, the boundary condition associated with surface force field $\mathbf{f}$ applied to the undeformed boundary $\partial \Omega_{S}$ is written as

$$
\mathbb{F} \mathbb{S} \mathbf{n}^{S}=\mathbf{f}
$$

The constitutive equation of the structure is chosen in the framework of the theory of the linear viscoelasticity in finite displacements [83] and is written using the hypothesis of instantaneous memory (see [84], references included),

$$
\mathbb{S}_{i j}=a_{i j \ell m}(\mathbf{x}) \mathbb{E}_{\ell m}+b_{i j \ell m}(\mathbf{x}) \frac{d \mathbb{E}_{\ell m}}{d t}
$$

in which the classical convention for summations over repeated Latin indices is used, where tensors $a_{i j \ell m}(\mathbf{x})$ and $b_{i j \ell m}(\mathbf{x})$ are fourth-order real tensors depending on $\mathbf{x}$, which verify symmetry and positiveness properties, and where $\mathbb{E}_{\ell m}$ is the Green-Lagrange strain tensor, which is defined by

$$
\begin{aligned}
\mathbb{E}_{\ell m}(\mathbf{x}, t) & =\frac{1}{2}\left(\mathbb{F}_{k \ell}(\mathbf{x}, t) \mathbb{F}_{k m}(\mathbf{x}, t)-\delta_{\ell m}\right) \\
& =\frac{1}{2}\left(\frac{\partial u_{\ell}}{\partial x_{m}}+\frac{\partial u_{m}}{\partial x_{\ell}}+\frac{\partial u_{k}}{\partial x_{\ell}} \frac{\partial u_{k}}{\partial x_{m}}\right) .
\end{aligned}
$$

\section{Boundary value problem in $(p, \eta, u)$}

For the linear dissipative acoustic liquid, the equations of motion are written in the reference configuration. Let $p(\mathbf{x}, t)$ be the acoustic pressure field defined in $\bar{\Omega}_{L}=\Omega_{L} \cup \partial \Omega_{L}$ (reference configuration), and let $\eta(\mathbf{x}, t)$ be the field defined on 
$\Gamma$, which is the normal displacement to the free surface $\Gamma$ along $\mathbf{n}$ at point $\mathbf{x}$ in the reference configuration. At time $t$, a surface force field and a body force field are applied to the structure in the actual configuration, which are transported on the reference configuration and give the force field $\mathbf{f}(\mathbf{x}, t)$ on $\Gamma_{E}$ (see Eq. (6)) and the body force field $\mathbf{b}(\mathbf{x}, t)$ in $\Omega_{S}$ (see Eq. (5)).

At time $t$, and in the reference configuration, the boundary value problem is expressed in terms of the structural displacement field $\mathbf{u}(\mathbf{x}, t)$, the internal pressure field $p(\mathbf{x}, t)$, and the normal displacement $\eta(\mathbf{x}, t)$ of the free surface. Under Cauchy initial conditions at time $t_{0}$, the problem consists in finding, for $t>t_{0}$, the fields $\mathbf{u}(\mathbf{x}, t), p(\mathbf{x}, t)$, and $\eta(\mathbf{x}, t)$, such that

$$
\begin{gathered}
\frac{1}{\rho_{0} c_{0}^{2}} \frac{\partial^{2} p}{\partial t^{2}}-\frac{\tau}{\rho_{0}} \nabla^{2} \frac{\partial p}{\partial t}-\frac{1}{\rho_{0}} \nabla^{2} p=0 \quad \text { in } \quad \Omega_{L}, \\
\left(1+\tau \frac{\partial}{\partial t}\right) \frac{\partial p}{\partial \mathbf{n}}=-\rho_{0} \frac{\partial^{2} \mathbf{u}}{\partial t^{2}} \cdot \mathbf{n} \quad \text { on } \quad \Gamma_{L}, \\
\left(1+\tau \frac{\partial}{\partial t}\right) \frac{\partial p}{\partial \mathbf{n}}=-\rho_{0} \frac{\partial^{2} \eta}{\partial t^{2}} \quad \text { on } \quad \Gamma, \\
p=\rho_{0} \eta \mathbf{g} \cdot \mathbf{n}-\sigma_{\Gamma}\left\{\left(\frac{1}{R_{1}^{2}}+\frac{1}{R_{2}^{2}}\right) \eta+\nabla_{\Gamma}^{2} \eta\right\} \quad \text { on } \quad \Gamma, \\
\frac{\partial \eta}{\partial \boldsymbol{\nu}}=c_{\eta} \eta+\mathcal{J}_{\mathbf{u}} \quad \text { on } \quad \gamma, \\
\rho_{\mathrm{s}} \frac{\partial^{2} \mathbf{u}}{\partial t^{2}}-\operatorname{div}(\mathbb{F} \mathbb{S})=\mathbf{b}, \forall \mathbf{x} \in \Omega_{S}, \\
\mathbb{F} \mathbb{S}^{S}=\mathbf{f} \quad \text { on } \quad \Gamma_{E}, \\
\mathbb{F} \mathbb{S}^{S} d \Gamma_{L}=-p \mathbf{n}^{S} d \Gamma_{L}+\sigma_{\Gamma}\left(\mathcal{J}^{\prime} \eta\right) d \mu_{\gamma} \text { on } \Gamma_{L},
\end{gathered}
$$

in which $\mathbb{F}$ is defined by Eq. (2) and where $\mathbb{S}$ is related to $\mathbb{E}$ (defined by Eq. (9)) by the constitutive equation defined by Eq. (8). In these equations, the different quantities are defined as follows:

- Eq. (10) is the linear dissipative acoustic equation, in which $\rho_{0}$ is the constant mass density of the homogeneous liquid at equilibrium, $c_{0}$ is the constant speed of sound, and $\tau$ is the constant coefficient that characterizes the dissipation in the 
internal liquid (as a function of the dynamic, kinematic, and second viscosities).

- Eq. (11) is the kinematic fluid-structure coupling condition on $\Gamma_{L}$, which expresses the continuity of the normal velocity field on the interface.

- Eq. (12) represents the kinematic equation for the free surface $\Gamma$.

- Eq. (13) corresponds to the free-surface constitutive equation of surface $\Gamma$, in which $\sigma_{\Gamma}$ is the surface tension coefficient, $\mathbf{g}$ is the gravitational acceleration vector, $R_{1}$ and $R_{2}$ are the principal curvature radii, and where $\nabla_{\Gamma}^{2} \eta$ denotes the surface Laplacian related to surface $\Gamma$ (if capillarity is neglected, the classical sloshing free-surface boundary condition is retrieved). It can be seen that $\left(1 / R_{1}^{2}+1 / R_{2}^{2}\right) \eta+\nabla_{\Gamma}^{2} \eta$ results from the change of the surface energy induced by an infinitesimal change in $\eta$, which yields a contribution to the surface pressure (classical Young-Laplace law) (see [13], Chapter 4).

- Eq. (14) correspond to a new boundary condition for the contact angle compatible with a deformable structure, considered here as linear viscoelastic with geometrical nonlinearities. In the right-hand side of Eq. (14), the first term corresponds to the classical contact angle condition on the contact line, $\gamma$, in which $c_{\eta}$ is the contact angle coefficient (which is only valid for fixed rigid structure) while the additional second term allows the structure deformation to be taken into account. In this term, $\mathcal{J}$ is a differential operator on manifold $\Gamma_{L}$, which is (i) defined on a set of sufficiently differentiable $\mathbb{R}^{3}$-valued functions that are the traces on $\Gamma_{L}$ of functions on $\Omega_{S}$, (ii) with values in a set of $\mathbb{R}$-valued functions that are defined on $\gamma$. A particular case for differential operator $\mathcal{J}$ is the one given in [13] (Section 4.3, page 80):

$$
\mathcal{J} \mathbf{u}=E \mathbf{u} \cdot \mathbf{n}^{S}-\frac{\partial\left(\mathbf{u} \cdot \mathbf{n}^{S}\right)}{\partial \boldsymbol{\nu}_{L}},
$$

in which $E$ is a real coefficient and where $\boldsymbol{\nu}_{L}$ is external unit normal to $\gamma$ belonging to the tangent plane to surface $\Gamma_{L}$. The physical interpretation of Eq. (18) corresponds to the change of the energy due to an infinitesimal change in the position of the contact line, which yields a change of the wetted surface.

- Eq. (17), which have been introduced for the first time in [1], corresponds to a new fluid-structure boundary condition on $\Gamma_{L}$ in the presence of capillarity, which allows the condition of contact angle to be taken into account in a presence of a 
linear viscoelastic structure with geometrical nonlinearities. For the readability of the present paper, a short summary of the construction is recalled hereinafter. The first term of the right-hand side corresponds to the fluid-structure coupling condition for a linear acoustic fluid coupled with the weak geometrical nonlinear structure. Concerning the second term, let $\mathbf{x} \mapsto f(\mathbf{x})$ be any real function defined on $\Gamma_{L}$ such that its trace on $\gamma$ is integrable on $\gamma$ with respect to the curvilinear measure $d \gamma$ on $\gamma$ (the length of curve $\gamma$ is $\int_{\gamma} d \gamma=|\gamma|$ ). Then, $d \mu_{\gamma}$ is a real measure on $\Gamma_{L}$ such that $\int_{\Gamma_{L}} f(\mathbf{x}) d \mu_{\gamma}(\mathbf{x})=\int_{\gamma} f(\mathbf{x}) d \gamma(\mathbf{x})$ (this means that the support of measure $d \mu_{\gamma}$ is $\gamma$ ). The term $\left(\mathcal{J}^{\prime} \eta\right) d \mu_{\gamma}$ is defined on $\Gamma_{L}$ by algebraic duality of the term $\mathcal{J} \mathbf{u}$ defined on $\gamma$ (see Eq. (14)) using the duality brackets,

$$
\begin{gathered}
<\mathcal{J} \mathbf{u}, \eta>_{d \gamma}=\int_{\gamma}(\mathcal{J} \mathbf{u}) \eta d \gamma, \\
\ll \mathbf{u}, \mathcal{J}^{\prime} \eta \gg_{d \mu_{\gamma}}=\int_{\Gamma_{L}}\left(\mathcal{J}^{\prime} \eta\right) \cdot \mathbf{u} d \mu_{\gamma} .
\end{gathered}
$$

The term $\left(\mathcal{J}^{\prime} \eta\right) d \mu_{\gamma}$ is then defined by

$$
\ll \mathbf{u}, \mathcal{J}^{\prime} \eta \gg_{d \mu_{\gamma}}=<\mathcal{J} \mathbf{u}, \eta>_{d \gamma} .
$$

\section{Computational fluid-structure model}

Let $\mathbf{P}(t), \mathbf{H}(t)$, and $\mathbf{U}(t)$ be the vectors corresponding to the spatial discretization of fields $p(\mathbf{x}, t), \eta(\mathbf{x}, t)$, and $\mathbf{u}(\mathbf{x}, t)$. The first time derivative is denoted by a dot and the second time derivative with a double dot. The discretization of the variational formulation of the boundary value problem in $(p, \eta, \mathbf{u})$ yields:

- for Eqs. (10) to (12),

$$
\left[\mathcal{A}^{L}\right]\{\mathbf{P}(t)\}-\left[C_{p \eta}\right]^{T} \ddot{\mathbf{H}}(t)-\left[C_{p \mathbf{u}}\right]^{T} \ddot{\mathbf{U}}(t)=0,
$$

in which $\left[\mathcal{A}^{L}\right]$ is the symmetric matrix-valued linear differential operator defined by $\left[\mathcal{A}^{L}\right]=[M] d^{2} / d t^{2}+[D] d / d t+[K]$.

- for Eqs. (13) and (14),

$$
\left[C_{p \eta}\right] \mathbf{P}(t)+\left(\left[K_{g}\right]+\left[K_{c}\right]\right) \mathbf{H}(t)+\left[C_{\eta \mathbf{u}}\right] \mathbf{U}(t)=0 .
$$

- for Eqs. (15) to (17),

$$
\begin{aligned}
{\left[C_{p \mathbf{u}}\right] \mathbf{P}(t)+\left[C_{\eta \mathbf{u}}\right]^{T} \mathbf{H}(t)+\left[M^{S}\right] \ddot{\mathbf{U}}(t) } & \\
& +\mathcal{A}^{S}\{\mathbf{U}(t), \dot{\mathbf{U}}(t)\}=\mathbf{F}^{S}(U(t), t),
\end{aligned}
$$


in which $\mathcal{A}^{S}$ is the vector-valued nonlinear differential operator that can be written as $\mathcal{A}^{S}\{\mathbf{U}(t), \dot{\mathbf{U}}(t)\}=\left[D^{S}(\mathbf{U}(t))\right] \dot{\mathbf{U}}(t)+\mathbf{K}^{S}\{\mathbf{U}(t)\}$, where $\left[D^{S}(\mathbf{U}(t))\right], \mathbf{K}^{S}\{\mathbf{U}(t)\}$ and $\mathbf{F}^{S}(\mathbf{U}(t), t)$ are nonlinear mappings of $\mathbf{U}(t)$. The different quantities introduced in Eqs. (22) to (24) are defined in Section 6.

It can be proved that, for all $t \geq t_{0}$, the problem defined by Eqs. (22) to (24) with the initial conditions, has a solution $(\mathbf{P}(t), \mathbf{H}(t), \mathbf{U}(t))$.

\section{Vectors and matrices of the discretized problem presented in Section 5}

In this section, we give the expressions of the vectors and matrices introduced in Section 5, which result from the discretization of the weak formulation of the boundary value problem defined by Eqs. (10) to (17). For such a construction, for fixed $t$, we consider the fields $(p(t), \eta(t), \mathbf{u}(t))$ and the test-function fields $(\delta p$, $\delta \eta, \delta \mathbf{u})$, which belong to the admissible spaces. In order to simplify the notations, parameter $t$ will be removed if there is no possible confusion.

Matrices related to the equations in P for the linear dissipative acoustic liquid.

- Symmetric real matrix $[M]$ is positive definite, and corresponds to the discretization of the bilinear form $\frac{1}{\rho_{0} c_{0}^{2}} \int_{\Omega_{L}} p \delta p d \mathbf{x}$.

- Symmetric real matrix $[K]$ is positive semidefinite with a kernel of dimension 1 , and corresponds to the discretization of the bilinear form $\frac{1}{\rho_{0}} \int_{\Omega_{L}} \nabla p \cdot \nabla \delta p d \mathbf{x}$.

- Symmetric real matrix $[D]=\tau[K]$ is positive semidefinite with a kernel of dimension 1.

Matrices related to the equations in $H$ for the liquid free surface with capillarity.

- Symmetric real matrix $\left[K_{g}\right]$ is positive definite, and corresponds to discretization of the bilinear form $\rho_{0} \int_{\Gamma} \mathbf{g} \cdot \mathbf{n} \eta \delta \eta d \Gamma$.

- Symmetric real matrix $\left[K_{c}\right]$ is positive definite, and corresponds to the discretization of the bilinear form $\sigma_{\Gamma} \int_{\Gamma} \nabla_{\Gamma} \eta \cdot \nabla_{\Gamma} \delta \eta d \Gamma-\sigma_{\Gamma} \int_{\Gamma}\left(\frac{1}{R_{1}^{2}}+\frac{1}{R_{2}^{2}}\right) \eta \delta \eta d \Gamma-$ $\sigma_{\Gamma} \int_{\gamma} c_{\eta} \eta \delta \eta d \gamma$

Matrices related to the coupling terms.

- Rectangular real matrix $\left[C_{p \mathbf{u}}\right]$ corresponds to the discretization of the bilinear form $-\int_{\Gamma_{L}} p \mathbf{n} \cdot \delta \mathbf{u} d \Gamma_{L}$ 
- Rectangular real matrix $\left[C_{p \eta}\right]$ corresponds to the discretization of the bilinear form $-\int_{\Gamma} p \delta \eta d \Gamma$.

- Rectangular real matrix $\left[C_{\eta \mathbf{u}}\right]$ corresponds to the discretization of the bilinear form $-\sigma_{\Gamma} \int_{\gamma}(\mathcal{J} \mathbf{u}) \delta \eta d \gamma$.

Vectors and matrices related to the equations in $\boldsymbol{u}$ for the structure with geometric nonlinearities and a linear viscoelastic constitutive equation without memory effects.

- Symmetric real matrix $\left[M^{S}\right]$ is positive definite, and corresponds to the discretization of the bilinear form $\int_{\Omega_{S}} \rho_{\mathrm{S}} \mathbf{u} \cdot \delta \mathbf{u} d \mathbf{x}$.

- The vector $\left[D^{S}(U(t))\right] \dot{U}(t)+K^{S}(U(t))$ corresponds to the discretization (see $[85,86,87,88,89])$ of $\int_{\Omega_{S}} \mathbb{F}_{i j} \mathbb{S}_{j k}\left(\partial \delta u_{i} / \partial x_{k}\right) d \mathbf{x}$, in which $\mathbb{F}$ is a function of $\mathbf{u}$ (see Eq. (2)) and where $\mathbb{S}$ is also a function of $\mathbf{u}$ (see Eqs. (8) and (9)).

Vector of external forces.

- Vector $F^{S}(U(t), t)$ of external forces correspond to the discretization of $\int_{\Gamma_{E}} \mathbf{f}$. $\delta \mathbf{u} d \Gamma_{E}+\int_{\Omega_{S}} \mathbf{b} \cdot \delta \mathbf{u} d \mathbf{x}$, in which $\mathbf{b}$ depends on $\mathbf{u}$ (see Eq. (5)) and where $\mathbf{f}$ also depends on $\mathbf{u}$ (see Eq. (6)).

\section{Decomposition of the admissible space of the discretized coupled problem and associated vector bases}

The method consists in constructing a decomposition of the admissible space $C_{P, H, U}$ of the discretized problem defined by Eqs. (22) to (24) and in constructing an adapted vector basis that spans each admissible space.

It can be shown (see [1]) that this admissible space can be decomposed in the following direct sum,

$$
C_{P, H, U}=C_{P} \oplus C_{H} \oplus C_{U},
$$

in which each admissible space and its associated vector basis is defined hereinafter.

\subsection{Admissible space $C_{P}$ and vector basis}

The space $C_{P}$ is related to the discretized problem in $\mathbf{P}$ (see Eq. (22)) for which the liquid is a linear acoustic liquid (without the dissipative term) occupying domain $\Omega_{L}$, and for which we have the boundary condition $\partial p / \partial \mathbf{n}=0$ on $\Gamma_{L}$ and 
$p=0$ on $\Gamma \cup \gamma$ (i.e. there are no sloshing and no capillarity). Therefore, $C_{P}$ is the admissible space for the problem defined by $[M] \ddot{\mathbf{P}}(t)+[K] \mathbf{P}(t)=0$ with $\mathbf{P}(t)=0$ for all the degrees of freedom (DOFs) related to $\Gamma \cup \gamma$. A vector basis of $C_{P}$ can then be constructed as the acoustic modes, which are the eigenvectors of the generalized eigenvalue problem with constraints,

$$
[K] \mathbf{P}=\lambda_{L}[M] \mathbf{P},
$$

$$
\text { with } \mathbf{P}=0 \text { for the DOF related to } \Gamma \cup \gamma \text {. }
$$

Let $[\mathcal{P}]=\left[\mathbf{P}_{1} \ldots \mathbf{P}_{N_{L}}\right]$ be the rectangular real matrix whose $N_{L}$ columns are constituted of the eigenvectors associated with the $N_{L}$ first smallest positive eigenvalues.

\subsection{Admissible space $C_{H}$ and vector basis}

The space $C_{H}$ is related to the discretized problem in $\mathbf{H}$ for which the liquid is considered as an inviscid incompressible liquid, with sloshing and capillarity, and for which the boundary condition is $\partial p / \partial \mathbf{n}=0$ on $\Gamma_{L}$. Therefore, $C_{H}$ is the admissible space of the problem, $[K] \mathbf{P}(t)-\left[C_{p \eta}\right]^{T} \ddot{\mathbf{H}}(t)=0$ and $\left[C_{p \eta}\right] \mathbf{P}(t)+\left(\left[K_{g}\right]+\left[K_{c}\right]\right) \mathbf{H}(t)=0$. A vector basis of $C_{H}$ can then be constructed as the sloshing-capillarity modes of liquid occupying domain $\Omega_{L}$, which are the eigenvectors of the generalized eigenvalue problem with constraints,

$$
\begin{gathered}
{[K] \mathbf{P}+\lambda_{\Gamma}\left[C_{p \eta}\right]^{T} \mathbf{H}=0,} \\
{\left[C_{p \eta}\right] \mathbf{P}+\left(\left[K_{g}\right]+\left[K_{c}\right]\right) \mathbf{H}=0 .}
\end{gathered}
$$

Since the kernel of $[K]$ is equal to 1 , the elimination of $\mathbf{P}$ yields,

$$
\begin{gathered}
{\left[K_{g c}\right] \mathbf{H}=\lambda_{\Gamma}\left[M_{\Gamma}\right] \mathbf{H} .} \\
{[L] \mathbf{H}=0}
\end{gathered}
$$

in which $[L]$ is a real row matrix, and where $\left[K_{g c}\right]$ and $\left[M_{\Gamma}\right]$, under the constraints $[L] \mathbf{H}=0$, are positive-definite symmetric matrices that are constructed as a function of matrices $[K],\left[C_{p \eta}\right],\left[K_{g}\right]$ and $\left[K_{c}\right]$. For practical construction of these matrices, we refer the reader to Section 4.6 of Chapter 4 in Ref. [13].

Let $[\mathcal{H}]=\left[\mathbf{H}_{1} \ldots \mathbf{H}_{N_{\Gamma}}\right]$ be the rectangular real matrix whose $N_{\Gamma}$ columns are constituted of the eigenvectors associated with the $N_{\Gamma}$ first smallest strictly positive eigenvalues. 


\subsection{Admissible space $C_{U}$ and vector basis}

Since the structure has a nonlinear behavior, the construction is presented in two steps: definition of the admissible space $C_{U}$ and then construction of a vector basis.

\subsubsection{Definition of the admissible space $C_{U}$}

$C_{U}$ is the admissible space of the discretized problem in $\mathbf{U}$ (see Eq. (24)), related to the viscoelastic structure $\Omega_{S}$, coupled with the liquid considered as an inviscid incompressible liquid (without sloshing and capillarity) occupying domain $\Omega_{L}$, with the boundary condition $p=0$ on $\Gamma$. We then obtain the following discretized equations in (P, $\mathbf{U})$ deduced from Eqs. (22) and (24),

$$
\begin{gathered}
{[K] \mathbf{P}(t)-\left[C_{p \mathbf{u}}\right]^{T} \ddot{\mathbf{U}}(t)=0 \text { with } \mathbf{P}(t)=0 \text { on } \Gamma \cup \gamma,} \\
{\left[C_{p \mathbf{u}}\right] \mathbf{P}(t)+\left[M^{S}\right] \ddot{\mathbf{U}}(t)+\left[D^{S}(\mathbf{U}(t))\right] \dot{\mathbf{U}}(t)} \\
+\mathbf{K}^{S}\{\mathbf{U}(t)\}=\mathbf{F}^{S}(\mathbf{U}(t), t) .
\end{gathered}
$$

Using Eq. (32), $\mathbf{P}(t)$ can be eliminated in order to obtain an equation in $\mathbf{U}(t)$. Let $\left[K_{*}^{-1}\right]$ be the matrix such that the solution of $[K] \mathbf{P}=\mathbf{F}$ (in which $\mathbf{F}$ is an arbitrary vector) under the constraint $\mathbf{P}=0$ on $\Gamma \cup \gamma$, is written as $\mathbf{P}=\left[K_{*}^{-1}\right] \mathbf{F}$. Consequently, Eq. (32) yields $\mathbf{P}(t)=\left[K_{*}^{-1}\right]\left[C_{p \mathbf{u}}\right]^{T} \ddot{\mathbf{U}}(t)$. By substitution in (33), we obtain

$$
\begin{aligned}
&\left(\left[M^{S}\right]+\left[M_{A}\right]\right) \ddot{\mathbf{U}}(t)+\left[D^{S}(\mathbf{U}(t))\right] \dot{\mathbf{U}}(t) \quad \\
&+\mathbf{K}^{S}\{\mathbf{U}(t)\}=\mathbf{F}^{S}(\mathbf{U}(t), t),
\end{aligned}
$$

in which $\left[M_{A}\right]$ is a positive symmetric matrix (called the added mass matrix), which is written as

$$
\left[M_{A}\right]=\left[C_{p \mathbf{u}}\right]\left[K_{*}^{-1}\right]\left[C_{p \mathbf{u}}\right]^{T} .
$$

\subsubsection{Construction of a vector basis for $C_{U}$}

Two main approaches can be used for constructing the vector basis for $C_{U}$, represented by the rectangular matrix $[\mathcal{U}]=\left[\mathbf{U}_{1} \ldots \mathbf{U}_{N_{S}}\right]$ in which the $N_{S}$ columns are $N_{S}$ algebraically independent vectors that span a subspace of dimension $N_{S}$.

- The first one is the proper orthogonal decomposition (POD) approach, which does not require expertise in the selection of the vector basis, but which yields a basis that depends on the selected excitation forces, and which requires solving a nonlinear dynamical problem in high dimension. 
- The second one is the linear modes approach, which requires a very great expertise for selecting the linear modes that participate in the nonlinear response of the structure.

The reader will find in [90] an extensive, comprehensive, and detailed review concerning the construction of such a vector basis in structural dynamics for a structure with nonlinear geometric effects.

In the present paper, we propose to construct a vector basis of $C_{U}$ in using the nonlinear structure coupled with the liquid considered as an incompressible inviscid liquid in order to take into account the effects of added mass. It should be noted that we do not recommend to use the structure in vacuo (no added mass effect taken into account). Let us recall that the added-mass effects corresponding to compressible and incompressible fluids are distinct (see Chapters 5 and 8 in [13]) and see [91, 92] in which it is shown that the added-mass of a compressible flow does not admit a simple local-in-time decomposition. This is the reason why the added-mass effects have been introduced using an associated incompressible liquid. Let us remark that solely for the construction of the basis of $C_{U}$, the use of the POD for the complete fluid-structure problem defined by Eqs. (22) to (24) would not be computationally efficient, and in addition is not in the spirit of the method proposed for constructing the reduced-order model for the fluid-structure problem under consideration.

(i) - POD-based approach. The POD approach requires to solve the following evolution problem

$$
\begin{aligned}
\left(\left[M^{S}\right]+\left[M_{A}\right]\right) \ddot{\mathbf{U}}(t)+\left[D^{S}(\mathbf{U}(t))\right] \dot{\mathbf{U}}(t) & \\
& +\mathbf{K}^{S}\{\mathbf{U}(t)\}=\mathbf{F}^{S}(\mathbf{U}(t), t) \quad t>t_{0},
\end{aligned}
$$

with the initial conditions

$$
\mathbf{U}\left(t_{0}\right)=\mathbf{U}_{0} \quad, \quad \dot{\mathbf{U}}\left(t_{0}\right)=\mathbf{V}_{0} .
$$

A displacement snapshot matrix $\left[U_{\text {snap }}\right]$ is formed using the responses $\mathbf{U}\left(t_{1}\right), \ldots, \mathbf{U}\left(t_{n}\right)$ of Eqs. (36) and (37) at $n$ instants $t_{1}, \ldots, t_{n}$ (snapshots) with $n$ sufficiently large, in forming the real symmetric matrix

$$
\left[R_{\text {snap }}\right]=\frac{1}{n}\left[U_{\text {snap }}\right]^{T}\left[U_{\text {snap }}\right]
$$


and in computing the dominant eigensubspace (associated with the $N_{S}$ largest positive eigenvalues $\mu_{1} \geq \ldots \geq \mu_{N_{S}}>0$ ), represented by the rectangular matrix $[\mathcal{U}]=\left[\mathbf{U}_{1} \ldots \mathbf{U}_{N_{S}}\right]$, of the eigenvalue problem,

$$
\left[R_{\text {snap }}\right] \mathbf{U}_{\alpha}=\mu_{\alpha} \mathbf{U}_{\alpha} \quad, \quad \alpha=1, \ldots, N_{S} .
$$

(ii) - Use of the linear modes. With the linear modes approach, the vector basis is constituted of the elastic modes of the underlying linear structural system with the added mass, and is then constructed by solving the generalized eigenvalue problem (deduced from Eq. (34)),

$$
\left[K^{(1)}\right] \mathbf{U}=\lambda_{S}\left(\left[M^{S}\right]+\left[M_{A}\right]\right) \mathbf{U}
$$

in which

$$
\left[K^{(1)}\right]_{i j}=\left.\left\{\partial\left\{\mathbf{K}^{S}\{\mathbf{U}\}\right\}_{i} / \partial U_{j}\right\}\right|_{\mathbf{U}=0},
$$

which is the linear part of the nonlinear operator $\mathbf{U} \mapsto \mathbf{K}^{S}\{\mathbf{U}\}$. As explained before, the linear modes $\mathbf{U}_{1} \ldots \mathbf{U}_{N_{S}}$ have to be carefully selected because all the participating modes in the nonlinear responses must be included [90]. This means that the associated eigenvalues $0 \leq \lambda_{S, 1} \leq \ldots \leq \lambda_{S, N_{S}}$ (which are in increasing order) are not, in general, consecutive. It should be noted that the eigenvectors that correspond to zero eigenvalues are the rigid body modes of the underlying linear structural system with the added mass (and not, in general, the rigid body modes of the nonlinear fluid-structure system) .

Let $[\mathcal{U}]=\left[\mathbf{U}_{1} \ldots \mathbf{U}_{N_{S}}\right]$ be the rectangular real matrix whose $N_{S}$ columns are constituted of the selected eigenvectors.

\section{Nonlinear reduced-order computational model}

8.1. Methodology for constructing the nonlinear reduced-order computational model

The reduced-order model of order $\left(N_{L}, N_{\Gamma}, N_{S}\right)$ is obtained by projecting $(\mathbf{P}(t), \mathbf{H}(t), \mathbf{U}(t))$ on the vector bases constructed in Section 7 ,

$$
\begin{aligned}
\mathbf{P}(t) & =[\mathcal{P}] \mathbf{q}^{P}(t), \\
\mathbf{H}(t) & =[\mathcal{H}] \mathbf{q}^{H}(t), \\
\mathbf{U}(t) & =[\mathcal{U}] \mathbf{q}^{U}(t),
\end{aligned}
$$


and then in projecting Eqs. (22) to (24). The two linear matrix equations defined by Eqs. (22) and (23) can classically be projected without any particular difficulties. Concerning the nonlinear equation defined by Eq. (24), due to the nonlinearity in $\mathbf{U}(t)$, several approaches can be used for constructing the projection in a computational framework, that we briefly summarized hereinafter. The possible approaches can be classified in the class of the intrusive methods with respect to the commercial softwares and the class of the non-intrusive one.

(i) For the class of intrusive methods, two main approaches can be identified:

(i-1) The first one consists in implementing, at each time step $t$ required by the time integration scheme used, the direct computation of the reduced nonlinear force $[\mathcal{U}]^{T} \mathcal{A}^{S}\left\{[\mathcal{U}] \mathbf{q}^{U}(t),[\mathcal{U}] \dot{\mathbf{q}}^{U}(t)\right\}$ and in introducing a hyper reduction technique for reducing the numerical cost of the computation (see $[68,69,70,71]$ ).

(i-2) The second one consists in implementing the direct computation of the reduced nonlinear terms that result from an algebraic calculation of the different contributions of the projection of $\mathcal{A}^{S}\{\mathbf{U}(t), \dot{\mathbf{U}}(t)\}$,

$$
\begin{aligned}
{[\mathcal{U}]^{T} \mathcal{A}^{S}\left\{[\mathcal{U}] \mathbf{q}^{U}(t),[\mathcal{U}] \dot{\mathbf{q}}^{U}(t)\right\} } & =[\mathcal{U}]^{T}\left[D^{S}\left([\mathcal{U}] \mathbf{q}^{U}(t)\right)\right][\mathcal{U}] \dot{\mathbf{q}}^{U}(t) \\
& +[\mathcal{U}]^{T} \mathbf{K}^{S}\left\{[\mathcal{U}] \mathbf{q}^{U}(t)\right\}
\end{aligned}
$$

The component $\alpha$ of the reduced conservative term can be written as

$$
\begin{aligned}
\left\{[\mathcal{U}]^{T} \mathbf{K}^{S}\left\{[\mathcal{U}] \mathbf{q}^{U}(t)\right\}\right\}_{\alpha} & =\mathcal{K}_{\alpha \beta}^{(1)} q_{\beta}^{U}(t) \\
& +\mathcal{K}_{\alpha \beta \gamma}^{(2)} q_{\beta}^{U}(t) q_{\gamma}^{U}(t)+\mathcal{K}_{\alpha \beta \gamma \delta}^{(3)} q_{\beta}^{U}(t) q_{\gamma}^{U}(t) q_{\delta}^{U}(t)
\end{aligned}
$$

with summation on the repeated Greek indices. The component $\alpha$ of the reduced dissipative term can be written as

$$
\begin{aligned}
{[\mathcal{U}]^{T}\left[D^{S}\left([\mathcal{U}] \mathbf{q}^{U}(t)\right)\right][\mathcal{U}] } & \dot{\mathbf{q}}^{U}(t)=\frac{d}{d t}\left\{\mathcal{D}_{\alpha \beta}^{(1)} q_{\beta}^{U}(t)\right. \\
& \left.+\mathcal{D}_{\alpha \beta \gamma}^{(2)} q_{\beta}^{U}(t) q_{\gamma}^{U}(t)+\mathcal{D}_{\alpha \beta \gamma \delta}^{(3)} q_{\beta}^{U}(t) q_{\gamma}^{U}(t) q_{\delta}^{U}(t)\right\} .
\end{aligned}
$$

With such a direct intrusive approach, all the constant coefficients of the tensors in the right-hand sides of Eqs. (46) and (47) are calculated inside the software. 
For the conservative term defined by Eq. (46), such a direct implementation can be found in $[93,94]$ and can be extended without difficulty to the dissipative term defined by Eq. (47).

(ii) For the class of non-intrusive methods related to the calculation of the conservative part defined by Eq. (46), the approach consists in numerically identifying all the coefficients of the tensors that appear in the right-hand side of Eq. (46) by solving an inverse problem based on the computation of the nonlinear static responses of the structure in vacuo submitted to appropriate Dirichlet conditions related to the selected vectors $\mathbf{U}_{1}, \ldots \mathbf{U}_{N_{S}}$ of the vector basis. In [90], the reader will find a detailed review concerning such a numerical identification for all the coefficients of the tensors relative to the elastic part (see the right-hand side of Eq. (46)). Some applications of such a methodology can be found, for instance, in $[4,95,96]$. This approach could certainly be extended for identifying the coefficients of the tensors related to the dissipative part (see the right-hand side of Eq. (47)).

\subsection{Nonlinear reduced-order computational model for the fluid-struture system}

Using the methodology presented in Section 8.1, the following nonlinear matrix equation is obtained for the reduced-order computational model,

$$
\begin{aligned}
{\left[A_{\mathrm{FSI}}\right]\left[\begin{array}{c}
\ddot{\mathbf{q}}^{P}(t) \\
\ddot{\mathbf{q}}^{H}(t) \\
\ddot{\mathbf{q}}^{U}(t)
\end{array}\right]+\left[B_{\mathrm{FSI}}\right]\left[\begin{array}{c}
\dot{\mathbf{q}}^{P}(t) \\
\dot{\mathbf{q}}^{H}(t) \\
\dot{\mathbf{q}}^{U}(t)
\end{array}\right] } \\
+\left[C_{\mathrm{FSI}}\right]\left[\begin{array}{c}
\mathbf{q}^{P}(t) \\
\mathbf{q}^{H}(t) \\
\mathbf{q}^{U}(t)
\end{array}\right] \\
0 \\
0 \\
+\left[\begin{array}{c}
{[\mathcal{U}]^{T} \mathcal{A}^{S}\left\{[\mathcal{U}] \mathbf{q}^{U}(t),[\mathcal{U}] \dot{\mathbf{q}}^{U}(t)\right\}} \\
0 \\
0 \\
0
\end{array}\right],
\end{aligned}
$$


in which the matrices $\left[A_{\mathrm{FSI}}\right],\left[B_{\mathrm{FSI}}\right]$, and $\left[C_{\mathrm{FSI}}\right]$ are written as

$$
\begin{gathered}
{\left[A_{\mathrm{FSI}}\right]=\left[\begin{array}{ccc}
{[\mathcal{M}]} & -\left[\mathcal{C}_{p \eta}\right]^{T} & -\left[\mathcal{C}_{p u}\right]^{T} \\
0 & 0 & 0 \\
0 & 0 & {\left[\mathcal{M}^{S}\right]}
\end{array}\right]} \\
{\left[B_{\mathrm{FSI}}\right]=\left[\begin{array}{ccc}
{[\mathcal{D}]} & 0 & 0 \\
0 & 0 & 0 \\
0 & 0 & 0
\end{array}\right]} \\
{\left[C_{\mathrm{FSI}}\right]=\left[\begin{array}{ccc}
{[\mathcal{K}]} & 0 & 0 \\
{\left[\mathcal{C}_{p \eta}\right]} & {\left[\mathcal{K}_{g c}\right]} & {\left[\mathcal{C}_{\eta u}\right]} \\
{\left[\mathcal{C}_{p u}\right]} & {\left[\mathcal{C}_{\eta u}\right]^{T}} & 0
\end{array}\right]}
\end{gathered}
$$

with the following definitions for all the matrices in Eqs. (49) to (51):

- For the reduced matrices relative to the liquid, $[\mathcal{M}]=[\mathcal{P}]^{T}[M][\mathcal{P}],[\mathcal{D}]=$ $[\mathcal{P}]^{T}[D][\mathcal{P}]$, and $[\mathcal{K}]=[\mathcal{P}]^{T}[K][\mathcal{P}]$.

- For the reduced matrix relative to the free surface, $\left[\mathcal{K}_{g c}\right]=[\mathcal{H}]^{T}\left(\left[K_{g}\right]+\right.$ $\left.\left[K_{c}\right]\right)[\mathcal{H}]$.

- For the reduced matrix relative to the structure, $\left[\mathcal{M}^{S}\right]=[\mathcal{U}]^{T}\left[M^{S}\right][\mathcal{P}]$.

- For the reduced matrices relative to the coupling, $\left[\mathcal{C}_{p \eta}\right]=[\mathcal{H}]^{T}\left[C_{p \eta}\right][\mathcal{P}]$, $\left[\mathcal{C}_{p u}\right]=[\mathcal{U}]^{T}\left[C_{p u}\right][\mathcal{P}]$, and $\left[\mathcal{C}_{\eta u}\right]=[\mathcal{H}]^{T}\left[C_{\eta u}\right][\mathcal{U}]$.

\section{Conclusion}

In this paper, a nonlinear reduced-order computational model has been constructed for a linear viscoelastic structure with weak nonlinear geometrical effects, the structure being coupled with a linear acoustic liquid with sloshing and capillarity on the free surface. The model proposed is derived from the one used in fluid-structure interaction for linear systems, for which the analysis of the acoustic-sloshing-capillarity phenomena is efficient thanks to the use of a projection on the linear modes of the linear acoustic liquid and on the sloshing modes with capillarity. Concerning the construction of the vector basis for the structure, its is proposed to use a POD approach for the viscoelastic structure with weak nonlinear geometrical effects and taking into account the added mass induced by 
the liquid. The methodology for constructing the vector bases of the admissible sets and for obtaining the nonlinear ROM are detailed. The computational nonlinear ROM presented can directly be used for analysis the vibration of such fluid-structure systems using commercial finite element softwares for computing the vector bases and the projections.

\section{References}

[1] Ohayon R, Soize C. Vibration of structures containing compressible liquids wit surface tension and sloshing effects. Reduced-order model. Computational Mechanics 2015; 55(6):1071-1078.

[2] Kana DD, Lindholm US, Abramson HN. An experimental study of liquid instability in a vibrating elastic tank. Journal of Spacecraft 1966; 3(8):11831188 .

[3] Soize C. Stochastic Models of Uncertainties in Computational Mechanics. American Society of Civil Engineers (ASCE), Reston, 2012.

[4] Mignolet MP, Soize C. Stochastic reduced order models for uncertain nonlinear dynamical systems, Computer Methods in Applied Mechanics and Engineering 2008; 197(45-48): 3951-3963.

[5] Ohayon R, Soize C. Advanced Computational Vibroacoustics - ReducedOrder Models and Uncertainty Quantification. Cambridge University Press, New York, 2014.

[6] de Gennes PG, Brochard-Wyart F, Quéré D. Capillarity and Wetting Phenomena. Drops, Bubbles, Pearls, Waves, Springer, New York, 2004.

[7] Landau L, Lifchitz E. Fluid Mechanics. Pergamon Press, Oxford, 1992.

[8] Abramson HN. The dynamic behavior of liquids in moving containers. NASA SP-106, 1966.

[9] Myshkis AD, Babskii VG, Kopachevskii ND, Slobozhanin LA, Tyuptsov AD, Wadhwa RS. Low-Gravity Fluid Mechanics. Springer-Verlag, Berlin, 1987.

[10] Dodge FT. The New "Dynamical Behaviour of Liquids in Moving Containers". Southwest Research Institute, San Antonio, Texas, 2000. 
[11] Moiseyev NM, Rumyantsev VV. Dynamic Stability of Bodies Containing Fluid. Volume 6, Applied Physics and Engineering Edition, Springer, New York, 1968.

[12] Ibrahim R. Liquid Sloshing Dynamics: Theory and Applications. Cambridge University Press, Cambridge, 2005.

[13] Morand HJP, Ohayon R. Fluid Structure Interaction. John Wiley \& Sons, Chichester, 1995.

[14] Bermùdez A, Rodríguez R, Santamarina D. Finite element computation of sloshing modes in containers with elastic baffle plates. International Journal for Numerical Methods in Engineering 2003; 56(3):447-467.

[15] Ohayon R. Reduced models for fluid-structure interaction problems. International Journal of Numerical Methods in Engineering 2004; 60(1):139-152.

[16] Felippa CA, Park KC, Ross MR. A classification of interface treatments for FSI, pp. 27-51 in Fluid Structure Interaction II, Springer, Berlin, 2010.

[17] Farhat C, Chiu EKY, Amsallem D, Schotté JS, Ohayon R. Modeling of fuel sloshing and its physical effects on flutter. AIAA Journal 2013; 51(9):22522265 .

[18] Schotté JS, Ohayon R. Linearized formulation for fluid-structure interaction: Application to the linear dynamic response of a pressurized elastic structure containing a fluid with a free surface. Journal of Sound and Vibration 2013; 332:2396-2414.

[19] Dettmer W, Perić D. A computational framework for free surface fluid flows accounting for surface tension. Computer Methods in Applied Mechanics and Engineering 2006; 195:3038-3071.

[20] Veldman AEP, Gerrits J, Luppes R, Helder JA, Vreeburg JPB. The numerical simulation of liquid sloshing on board spacecraft. Journal of Computational Physics 2007; 224:82-99.

[21] El-Kamali M, Schotté JS, Ohayon R. Three-dimensional modal analysis of sloshing under surface tension. International Journal for Numerical Methods in Fluids 2011; 65:87-105. 
[22] Pukhnachev VV, Solonnikov VA. On the problem of dynamic contact angle. Journal of Applied Mathematics and Mechanics 1982; 46:961-971.

[23] Concus P, Finn R. On the behavior of a capillarity surface in a wedge. Proceedings of the National Academy of Sciences 1969; 63(2):292-299.

[24] Thompson PA, Robbins MO. Simulation of contact line motion: slip and the dynamic contact angle. Physical Review Letters 1989; 63:766-769.

[25] Cocciaro B, Faetti S, Nobili M. Capillarity effects on the surface gravity waves in a cylindrical container: wetting boundary conditions. Journal of Fluid Mechanics 1991; 231:325-343.

[26] Dussan V, Ramé E, Garoff S. On identifying the appropriate boundary conditions at moving contact line: an experimental investigation. Journal of Fluid Mechanics 1991; 230:97-111.

[27] Keller JB, Merchant G. Flexural rigidity of a liquid surface. Journal of Statistical Physics 1991; 63:1039-1051.

[28] Henderson DM, Miles JW. Surface-wave damping in a circular cylinder with a fixed contact line. Journal of Fluid Mechanics 1994; 275:285-299.

[29] Shankar PN, Kidambi R. The contact angle in inviscid fluid mechanics. Proceedings of the Indian Academy of Sciences (Math. Sci.) 2005; 115(2):227240 .

[30] Miras T, Schotté JS, Ohayon R. Energy approach for static and linearized dynamic studies of elastic structures containing incompressible liquids with capillarity: a theoretical formulation. Computational Mechanics 2012; 50:729741.

[31] Finn R. On the equations of capillary. Journal of Mathematical Fluid Mechanics 2001; 3:139-151.

[32] Finn R. The contact angle in capillarity. Physics of Fluids 2006; 18:047102.

[33] Finn R, Luli GK. On the capillary problem for compressible fluids. Journal of Mathematical Fluid Mechanics 2007; 9:87-103.

[34] Luke JC. A variational principle for a fluid with a free surface. Journal of Fluid Mechanics 1967; 27(2):395-397; 
[35] Miles JW. Nonlinear surface-waves in closed basins. Journal of Fluid Mechanics 1976; 75:419-448.

[36] Limarchenko OS. Effect of capillarity on the dynamics of a container liquid system. Soviet Applied Mechanics 1981, 17(6):601-604.

[37] Limarchenko OS. Application of the variational method to the solution of nonlinear problems of the dynamics of combined motions of a tank with fluid. Soviet Applied Mechanics 1983, 19(11):1021-1025.

[38] Peterson LD, Crawley EF, Hansman RJ. Nonlinear fluid slosh coupled with a dynamics of a spacecraft. AIAA Journal 1989; 27(9):1230-1240.

[39] Harari I, Grosh K, Hughes TJR, Malhotra M, Pinsky PM, Stewart JR, Thompson LL. Recent development in finite element methods for structural acoustics. Archives of Computational Methods in Engineering 1996; 3(23):131-309.

[40] Ohayon R, Soize C. Structural Acoustics and Vibration. Academic Press, London, 1998.

[41] Ohayon R, Soize C. Advanced computational dissipative structural acoustics and fluid-structure interaction in low- and medium-frequency domains. Reduced-order models and uncertainty quantification. International Journal of Aeronautical and Space Sciences 2012; 13(2):127-153.

[42] Soize C. Coupling between an undamped linear acoustic fluid and a damped nonlinear structure - Statistical energy analysis considerations. Journal of the Acoustical Society of America 1995; 98(1):373-385.

[43] Tezduyar T, Behr M, Liou J. A new strategy for finite element computations involving moving boundaries and interfaces - The deforming-spatialdomain/space-time procedure: I. The concept and preliminary numerical tests. Computer Methods in Applied Mechanics and Engineering 1992; 94:339-351.

[44] Tezduyar T, Behr M, Mittal S, Liou J. A new strategy for finite element computations involving moving boundaries and interfaces - The deformingspatial-domain/space-time procedure: II. Computation of free-surface flows, two-liquid flows, and flows with drifting cylinders. Computer Methods in Applied Mechanics and Engineering 1992; 94:353-371. 
[45] Farhat C, M. Lesoinne M, Le Tallec P. Load and motion transfer algorithms for fluid/structure interaction problems with non-matching discrete interfaces: Momentum and energy conservation, optimal discretization and application to aeroelasticity. Computer Methods in Applied Mechanics and Engineering 1998; 157(1-2):95-114.

[46] Farhat C, Geuzaine P, Brown G. Application of a three-field nonlinear fluidstructure formulation to the prediction of the aeroelastic parameters of an F-16 fighter. Computers and Fluids 2003; 32(1):3-29.

[47] Tezduyar T. Interface-tracking and interface-capturing techniques for finite element computation of moving boundaries and interfaces. Computer Methods in Applied Mechanics and Engineering 2006; 195:2983-3000.

[48] Wall WA, Genkinger S, Ramm E. A strong coupling partitioned approach for fluid-structure interaction with free surfaces. Computers and Fluids 2007; 36(1): 169-183

[49] Bazilevs Y, Calo VM, Hughes TJR, Zhang Y. Isogeometric fluid-structure interaction: theory, algorithms, and computations. Computational Mechanics 2008; 43(1):3-37.

[50] Takizawa K, Tezduyar TE. Multiscale space-time fluid-structure interaction techniques. Computational Mechanics 2011; 48:247-267.

[51] Bazilevs Y, Takizawa K, Tezduyar TE. Computational Fluid-Structure Interaction. John Wiley \& Sons, Chichester, 2013.

[52] Nobile F, Pozzoli M, Vergara C. Time accurate partitioned algorithms for the solution of fluid-structure interaction problems in haemodynamics. Computers and Fluids 2013; 86:470-482

[53] Farhat C, Lakshminarayana VK. An ALE formulation of embedded boundary methods for tracking boundary layers in turbulent fluid-structure interaction problems. Journal of Computational Physics 2014; 263:53-70.

[54] Li Z, Leduc J, Combescure A, Leboeuf F. Coupling of SPH-ALE method and finite element method for transient fluid-structure interaction. Computers and Fluids 2014; 103:6-17. 
[55] Becker P, Idelsohn SR, Oñate, E. A unified monolithic approach for multifluid flows and fluid-structure interaction using the Particle Finite Element Method with fixed mesh 2015; 55(6):1091-1104.

[56] Cahn JW, Hilliard JE. Free energy of a nonuniform system. I. Interfacial free energy. The Journal of Chemical Physics 1958; 28(2):258-267.

[57] van Brummelen EH, Shokrpour-Roudbari M, van Zwieten GJ. Elastocapillarity simulations based on the Navier-Stokes-Cahn-Hilliard equations. ArXiv 1510.02441v1 2015; 1-8.

[58] Lieu T, Farhat C, Lesoinne M. Reduced-order fluid/structure modeling of a complete aircraft configuration. Computer Methods in Applied Mechanics and Engineering 2006; 195(41-43):5730-5742.

[59] Grepl MA, Maday Y, Nguyen NC, Patera A. Efficient reduced-basis treatment of nonaffine and nonlinear partial differential equations. ESAIM: Mathematical Modelling and Numerical Analysis 2007; 41(03):575-605.

[60] Nguyen N, Peraire J. An efficient reduced-order modeling approach for nonlinear parametrized partial differential equations. International Journal for $\mathrm{Nu}$ merical Methods in Engineering 2008; 76(1):27-55.

[61] Amsallem D, Cortial J, Carlberg K, Farhat C. A method for interpolating on manifolds structural dynamics reduced order models. International Journal for Numerical Methods in Engineering 2009; 80(9):1241-1258.

[62] Carlberg K, Bou-Mosleh C, Farhat C. Efficient non-linear model reduction via a least-squares Petrov-Galerkin projection and compressive tensor approximations. International Journal for Numerical Methods in Engineering 2011; 86(2):155-181.

[63] Amsallem D, Zahr MJ, Farhat C. Nonlinear model order reduction based on local reduced-order bases. International Journal for Numerical Methods in Engineering 2012; 92(10):891-916.

[64] Amsallem D, Farhat C. On the stability of projection-based linear reducedorder models: Descriptor vs nondescriptor forms, pp. 215-234 in Reduced Order Methods for Modeling and Computational Reduction. Springer MS \& A Series, 2014. 
[65] Farhat C, Avery P, Chapman T, Cortial J. Dimensional reduction of nonlinear finite element dynamic models with finite rotations and energy-based mesh sampling and weighting for computational efficiency. International Journal for Numerical Methods in Engineering 2014; 98(9):625-662.

[66] Hetmaniuk U, Tezaur R, Farhat C. Review and assessment of interpolatory model order reduction methods for frequency response structural dynamics and acoustics problems. International Journal for Numerical Methods in Engineering 2012; 90:1636-1662.

[67] Hetmaniuk U, Tezaur R, Farhat C. An adaptive scheme for a class of interpolatory model reduction methods for frequency response problems. International Journal for Numerical Methods in Engineering 2013; 93:1109-1124.

[68] Bui-Thanh T, Murali D, Willcox K. Proper orthogonal decomposition extensions for parametric applications in compressible aerodynamics. AIAA $\mathrm{Pa}$ per 2003-4213, 21 st Applied Aerodynamics Conference, Orlando, Florida, June 23-26, 2003.

[69] Ryckelynck D. A priori hyperreduction method: an adaptive approach. Journal of Computational Physics 2005; 202:346-366.

[70] Amsallem D, Zahr M, Choi Y, Farhat C. Design optimization using hyperreduced-order models. Structural and Multidisciplinary Optimization 2015; 51(4):919-940.

[71] Farhat C, Chapman T, Avery P. Structure-preserving, stability, and accuracy properties of the Energy-Conserving Sampling and Weighting (ECSW) method for the hyper reduction of nonlinear finite element dynamic models. International Journal for Numerical Methods in Engineering 2015; 102(5):1077-1110.

[72] Holmes P, Lumley J, Berkooz G. Turbulence, Coherent Structures, Dynamical Systems and Symmetry. Cambridge University Press: Cambridge, 1996.

[73] Soize C. Reduced models in the medium frequency range for general dissipative structural-dynamics systems. European Journal of Mechanics - A/Solids 1998; 17(4):657-685.

[74] Han S, Feeny BF. Enhanced proper orthogonal decomposition for the modal analysis of homogeneous structures. Journal of Vibration and Control 2002; 8(1):19-40. 
[75] Amabili M, Sarkar A, Paidoussis MP. Reduced-order models for nonlinear vibrations of cylindrical shells via the proper orthogonal decomposition method. Journal of Fluids and Structures 2003; 18(2):227-250.

[76] Kerschen G, Golinval JC, Vakakis AF, Bergman LA. The method of proper orthogonal decomposition for dynamical characterization and order reduction of mechanical systems: an overview. Nonlinear Dynamics 2005; 41:147-169.

[77] Sampaio R, Soize C. Remarks on the efficiency of POD for model reduction in nonlinear dynamics of continuous elastic systems. International Journal for Numerical Methods in Engineering 2007; 72(1):22-45.

[78] Willcox K, Peraire J. Balanced model reduction via the proper orthogonal decomposition. AIAA Journal 2002; 40(11):2323-2330.

[79] Prudhomme C, Rovas D, Veroy K, Machiels L, Maday Y, Patera A, Turinici G. Reliable real-time solution of parametrized partial differential equations: Reduced-basis output bound methods. Journal of Fluids EngineeringTransactions of the ASME 2002; 124(1):70-80.

[80] Astrid P, Weiland S, Willcox K, Backx T. Missing point estimation in models described by proper orthogonal decomposition. IEEE Transactions on Automatic Control 2008; 53(10):2237-2251.

[81] Ciarlet PG. Mathematical Elasticity: Three-dimensional elasticity, Volume 1. Elsevier, Amsterdam, 1993.

[82] Fung YC, Tong P. Classical and Computational Solid Mechanics. World Scientific, Singapore, 2001.

[83] Coleman BD, Noll W. Foundations of linear viscoelasticity. Review of Modern Physics 1961; 33(2):239249.

[84] Desceliers C, Soize C. Nonlinear viscoelastodynamic equations of threedimensional rotating structures in finite displacement and finite element discretization. International Journal of Non-linear Mechanics 2004; 39(3): 343368 .

[85] Zienkiewicz OC, Taylor RL. The Finite Element Method for Solid and Structural Mechanics. Sixth edition. Elsevier, Butterworth-Heinemann, Amsterdam, 2005 . 
[86] Bonet J, Wood RD. Nonlinear Continuum Mechanics for Finite Element Analysis. Second edition. Cambridge University Press, Cambridge, 2008.

[87] Wriggers P. Nonlinear Finite Element Methods. Springer-Verlag, Berlin, 2010.

[88] De Borst R, Crisfield MA, Remmers JJC, Verhoosel CV. Nonlinear Finite Element Analysis of Solids and Structures. Second edition. John Wiley \& Sons, Chichester, 2012.

[89] Belytschko T, Liu WK, Moran B, Elkhodary K. Nonlinear Finite Elements for Continua and Structures. John Wiley \& Sons, Chichester, 2014.

[90] Mignolet MP, Przekop A, Rizzi SA, Spottswood SM. A review of indirect/non-intrusive reduced order modeling of nonlinear geometric structures. Journal of Sound and Vibration 2013; 332:2437-2460.

[91] van Brummelen EH. Added mass effects of compressible and incompressible flows in fluid-structure interaction. Journal of Applied Mechanics 2009; 76:021206-1-7.

[92] van Brummelen EH. Partitioned iterative solution methods for fluid-structure interaction. International Journal for Numerical Methods in Fluids 2011; $65: 327$.

[93] Capiez-Lernout E, Soize C, Mignolet MP. Post-buckling nonlinear static and dynamical analyses of uncertain cylindrical shells and experimental validation. Computer Methods in Applied Mechanics and Engineering 2014; 271(1):210230 .

[94] Capiez-Lernout E, Soize C, Mbaye M. Mistuning analysis and uncertainty quantification of an industrial bladed disk with geometrical nonlinearity. Journal of Sound and Vibration 2015; 356:124-143.

[95] Perez R, Wang XQ, Mignolet MP. Nonlinear reduced-order models for thermoelastodynamic response of isotropic and functionally graded panels. AIAA Journal 2011; 49(3):630-641.

[96] Murthy R, Wang XQ, Perez R, Mignolet MP, Richter LA. Uncertainty-based experimental validation of nonlinear reduced order models. Journal of Sound and Vibration 2012; 331:1097-1114. 